\title{
物理气相传输法生长碳化硅单晶原生表面形貌研究
}

\author{
崔涂心 ${ }^{1,2}$, 胡小波 ${ }^{3}$, 徐现刚 ${ }^{3}$
}

(1. 中国工程物理研究院微系统与太赫兹研究中心, 成都 $610200 ; 2$. 中国工程物理研究院 电子工程研究所, 绵 阳 621999; 3. 山东大学 晶体材料国家重点实验室, 济南 250100)

摘 要: 晶体的生长原生面在一定程度上能反应出晶体生长机制和晶体缺陷分布的丰富信息。采用激光共聚焦显微 镜偏光拼接技术和原子力显微镜对物理气相传输法生长的 $4 \mathrm{H} 、 6 \mathrm{H}-\mathrm{SiC}$ 单晶原生小面的表面形貌进行了观察和测 试。偏光显微镜和原子力显微镜测试结果显示 $4 \mathrm{H}-\mathrm{SiC}$ 原生小面扩展后, 其周边趋向于形成六边形的生长台阶; 而 $6 \mathrm{H}-\mathrm{SiC}$ 原生小面扩展后, 其周边趋向于形成圆形的生长台阶。基于 Jackson 双层界面模型, 从热力学角度计算了 $4 \mathrm{H} 、 6 \mathrm{H}-\mathrm{SiC}$ 单晶的 Jackson 因子 $\alpha$ 分别为 33.15 和 31.87 , 故导致 $4 \mathrm{H} 、 6 \mathrm{H}-\mathrm{SiC}$ 单晶原生小面台阶形貌差异的是生 长界面的粗糙程度和生长温度。原生面上的微管缺陷是生长台阶的起源, 借助原子力显微镜对多个微管进行了测 试。由测试结果可知, 微管直径分布在 $760 \mathrm{~nm}-6.0 \mathrm{um}$ 之间, 相应的伯格斯矢量绝对值分布在 $5 \mathrm{c} \sim 14 \mathrm{c}$, 微管直径与 伯格斯矢量平方值的商 $D / B^{2}$ 分布在 $11.1 \sim 23.6 \mathrm{~nm}^{-1}$ 之间, 即通过原子力显微镜测试获得的微管结构数据不严格遵 守 Frank 理论。

关 键 词: 碳化硅; 原生小面; 微管结构

中图分类号: O766 文献标识码: A

\section{As-grown Surface Morphologies of SiC Single Crystals Grown by PVT Method}

\author{
CUI Ying-Xin ${ }^{1,2}$, HU Xiao-Bo ${ }^{3}$, XU Xian-Gang ${ }^{3}$
}

(1. Microsystem and Terahertz Research Center, China Academy of Engineering Physics, Chengdu 610200, China; 2. Institute of Electronic Engineering, China Academy of Engineering Physics, Mianyang 621999, China; 3. State Key Laboratory of Crystal Materials, Shandong University, Jinan 250100, China)

\begin{abstract}
As-grown surface of single crystal can reflect abundant information including crystal growth mechanism and defect distribution of the single crystal after growth. The as-grown surface morphologies of $4 \mathrm{H}-\mathrm{SiC}$ and $6 \mathrm{H}-\mathrm{SiC}$ single crystals were observed and measured by laser confocal microscope DIC splicing technique and atomic force microscope (AFM). It is found that as-grown $4 \mathrm{H}-\mathrm{SiC}$ surface tends to appear hexagonal growth steps, while the as-grown $6 \mathrm{H}-\mathrm{SiC}$ surface tends to appear circular growth steps. Based on Jackson model and thermodynamics theory, Jackson factors of the $4 \mathrm{H}-\mathrm{SiC}$ and $6 \mathrm{H}-\mathrm{SiC}$ single crystals are calculated to be 33.15 and 31.87 , respectively. Therefore, the surface morphology difference between $4 \mathrm{H}-\mathrm{SiC}$ and $6 \mathrm{H}-\mathrm{SiC}$ is caused by the roughness of growth interface and crystal growth temperature. The micropipe on the as-grown surface is the origin of growth steps and multiple micropipes on the as grown surface were measured by AFM. Their diameters are in the range of $760 \mathrm{~nm}$ $6.0 \mu \mathrm{m}$, and the corresponding absolute values of Burgers vector are in the range of 5c-14c. Through structure characterization and statistical analysis, the values of micropipe diameters divided by squares of the burgers vectors
\end{abstract}

收稿日期：2017-10-30; 收到修改稿日期：2018-01-17

基金项目：科学挑战专题(TZ2018003) Science Challenge Project (TZ2018003)

作者简介: 崔涂心(1988-), 女, 助理研究员. E-mail: cuiyingxin@mtrc.ac.cn

通讯作者：胡小波, 教授. E-mail: xbhu@sdu.edu.cn 
$\left(D / B^{2}\right)$ are in the range of $11.1 \mathrm{~nm}^{-1}-23.6 \mathrm{~nm}^{-1}$, which means the structure data of these micropipes obtained by

AFM cannot strictly obey the Frank theory.

Key words: $\mathrm{SiC}$; as-grown surface; structure of micropipes

$\mathrm{SiC}$ 单晶材料具有宽禁带、高临界击穿电场、 高热导率、高载流子饱和漂移速度和极好的化学稳 定性等特点, 制造的器件可应用在高温和高频率、 高电压、大功率和强辐射等苛刻环境下, 在白光照 明、汽车电子化、雷达通信、石油钻井、航天航空、 核反应堆系统及军事装备等领域有着广泛的应用 ${ }^{[1-3]}$ 。

$\mathrm{SiC}$ 是一种天然超晶格, $\mathrm{SiC}$ 晶体具有 200 多种 同质多型体 ${ }^{[4]}$, 最常见只有 $3 \mathrm{C} 、 4 \mathrm{H} 、 6 \mathrm{H}$ 和 $15 \mathrm{R}$ 四 种晶型，目前可用的块体单晶祄底仅有 $4 \mathrm{H}-\mathrm{SiC}$ 和 $6 \mathrm{H}-\mathrm{SiC}$ 。采用物理气相传输法或升华法生长的 $\mathrm{SiC}$ 单晶的晶型控制依赖于生长温度和压力 ${ }^{[5]}$ 、温度梯 度 ${ }^{[6]}$ 和晶体生长的籽晶面 ${ }^{[7]}$ 等生长参数。Masatoshi 等 ${ }^{[5]}$ 通过 60 个单晶生长的实验得知, 与 $6 \mathrm{H}-\mathrm{SiC}$ 单 晶生长相比, 在低温低压的环境下更易获得稳定的 $4 \mathrm{H}-\mathrm{SiC}$ 单晶。Schulze ${ }^{[8]}$ 和 Straubinger ${ }^{[9]}$ 等采用不同 䊏晶的不同生长面进行生长表明, 只有采用 $4 \mathrm{H}-\mathrm{SiC}$ 碳面作籽晶才能获稳定的 $4 \mathrm{H}$ 晶型, 采用其他面作 为籽晶容易导致多型夹杂。综上所述, 一般通过调 节生长温度、压力、籽晶等参数来控制晶型。

晶体的生长原生面在一定程度上能反映出晶体 生长机制和晶体缺陷分布的丰富信息, 通过观察并 研究单晶的表面生长形貌, 有助于了解晶体的生长 机制; 同时将表面生长形貌与生长条件相联系, 对 优化生长条件进而得到高质量的单晶非常重要 ${ }^{[10]}$ 。 本工作使用激光共聚焦显微镜和原子力显微镜对物 理气相传输法生长的 $\mathrm{SiC}$ 单晶原生小面的表面形貌 进行了观察, 并对其呈现的形貌差异做了理论解释。

\section{1 实验方法}

采用物理气相传输法生长 $4 \mathrm{H}-\mathrm{SiC}$ 和 $6 \mathrm{H}-\mathrm{SiC}$ 单 晶, 单晶生长设备采用中频感应加热方式。 $4 \mathrm{H}-\mathrm{SiC}$ 单晶用籽晶 $(000 \overline{1}) \mathrm{C}$ 面做生长面, 而 $6 \mathrm{H}-\mathrm{SiC}$ 单晶用 籽晶(0001)Si 面做生长面。在单晶生长过程中, 生长 腔内典型的压力与籽晶温度分别控制在 $10^{2} \sim 10^{4} \mathrm{~Pa}$ 和 $2000 \sim 2250^{\circ} \mathrm{C}$ 范围。采用 LEXT 激光共聚焦显微 镜观察晶体原生小面的生长台阶形貌, 其激光光源 为 $405 \mathrm{~nm}$, 水平分辨率和垂直分辨率分别为 0.12 和 $0.01 \mu \mathrm{m}$; 采用 Dimension Icon 原子力显微镜对原生 小面微管结构进行测试与表征, 其横向分辨率和纵 向分辨率分别为 0.1 和 $0.01 \mathrm{~nm}$ 。在观察和测试前,
先用 $8.104 \times 10^{5} \mathrm{~Pa}$ 压缩气体对晶体表面进行除尘处 理, 然后分别用优级丙酮、无水乙醇清洗表面。

\section{1 原生小面的生长台阶形貌}

从晶体生长的观点来看, 原生小面是由于生长 层的发展而形成的面, 随着晶体生长而延展, 总是 在晶体生长的终态表面上出现。由文献 ${ }^{[11-12]}$ 可知, $4 \mathrm{H}-\mathrm{SiC}$ 单晶采用籽晶 $(000 \overline{1}) \mathrm{C}$ 面做生长面、 $6 \mathrm{H}-\mathrm{SiC}$ 单晶用籽晶(0001) $\mathrm{Si}$ 面做生长面, 生长的晶体晶型 比较稳定, 故实验分别采用 $(000 \overline{1}) \mathrm{C}$ 面和(0001) $\mathrm{Si}$ 面做籽晶进行 $4 \mathrm{H} 、 6 \mathrm{H}-\mathrm{SiC}$ 单晶的生长，其原生小面 分别是 $(000 \overline{1}) \mathrm{C}$ 面和(0001)Si 面。

$4 \mathrm{H}-\mathrm{SiC}$ 单晶原生面的典型形貌如图 1 所示, 该 图由激光共聚焦显微镜的偏光拼接技术获得。 $4 \mathrm{H}-\mathrm{SiC}$ 单晶用籽晶 $(000 \mathrm{~T}) \mathrm{C}$ 面做生长面, 其原生面 中心由六角形分布的台阶包围, 六边形的边平行于 [11 20$]$ 方向且其长度约为 $2 \mathrm{~mm}$, 即生长台阶的形状 体现了晶体学的六次对称性, 且 $4 \mathrm{H}-\mathrm{SiC}$ 单晶的 [0001]轴为其六重轴。其他 4 H-SiC 单晶的原生面同 样呈现六边形或近六边形的台阶形貌, 如图 2 所示。

$6 \mathrm{H}-\mathrm{SiC}$ 单晶原生面的典型形貌如图 3 所示。 $6 \mathrm{H}-\mathrm{SiC}$ 单晶用籽晶(0001) Si 面做生长面, 其原生面 中心由近似圆形分布的台阶包围, 即生长台阶体现 出晶体学各向同性的特点。

其他 $6 \mathrm{H}-\mathrm{SiC}$ 单晶的原生面同样呈现近似圆形 分布的台阶形貌，如图 4 所示。

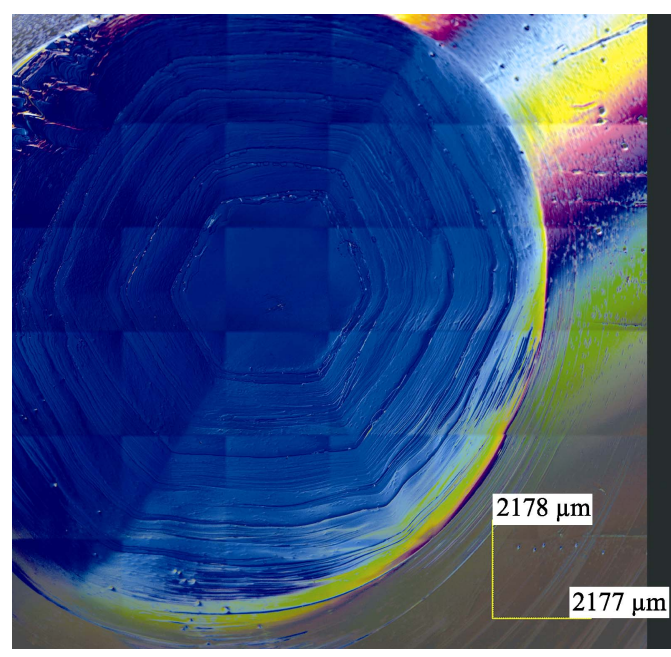

图 $14 \mathrm{H}-\mathrm{SiC}$ 单晶典型的原生面形貌

Fig. 1 Typical as-grown surface morphology of $4 \mathrm{H}-\mathrm{SiC}$ 


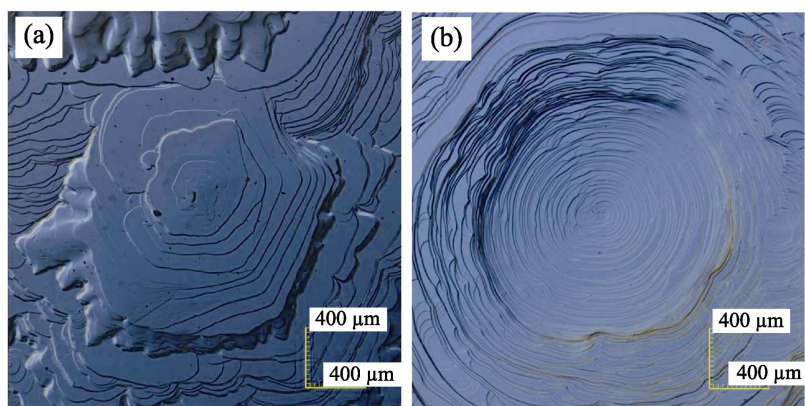

图 2 其他 4H-SiC 单晶原生面形貌

Fig. 2 Surface morphologies of the other as-grown 4H-SiC single crystal

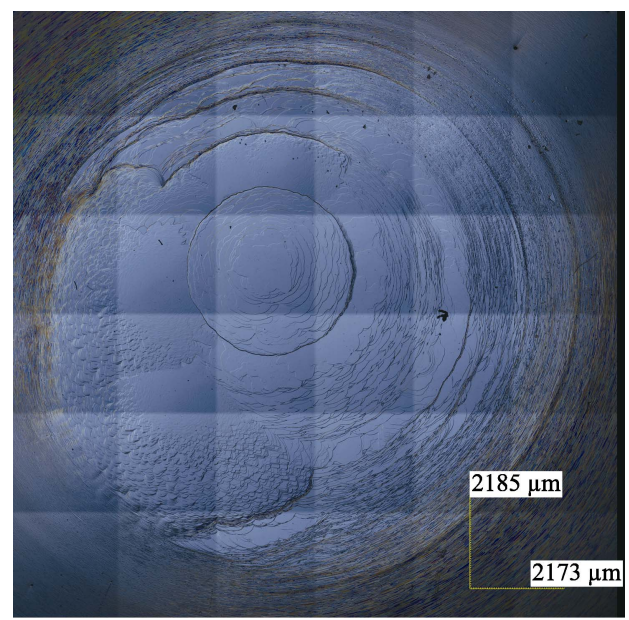

图 $36 \mathrm{H}-\mathrm{SiC}$ 单晶典型的原生面形貌

Fig. 3 Typical as-grown surface morphology of $6 \mathrm{H}-\mathrm{SiC}$

\section{2 原生小面的微管结构}

无论是 $4 \mathrm{H}-\mathrm{SiC}$ 还是 $6 \mathrm{H}-\mathrm{SiC}$ 单晶, 原生小面的 中心表面平坦, 斜率几乎为零。晶体表面隐约可见 微小凹坑, 这可能是晶体生长过程中粉末原料的分 解、升华对表面的撞击而成。以 $6 \mathrm{H}-\mathrm{SiC}$ 单晶为例, 通过 AFM 观察原生小面的形貌, 发现小面上分布 着均匀的生长台阶, 如图 5 所示, 这些生长台阶都
是从晶体缺陷微管处产生。根据 Frank 提出的微管 形成的螺位错机制 ${ }^{[13-14]}, \mathrm{SiC}$ 单晶中的微管是具有 较大伯格斯(Burgers)矢量的空心螺位错, 又称为超 级螺位错。由于与位错相关的应变能正比于 Burgers 矢量的平方，包含微管的晶体，通过去除位错的核 心来降低其应变能，因此微管的中心应为空心管。 微管直径 $D$ 与其伯格斯矢量大小 $B$ 之间存在如下定 量关系:

$$
D=\frac{\mu B^{2}}{4 \pi^{2} \gamma}
$$

其中: $D$ 为微管的直径 $(\mathrm{m}) ; \mu$ 为晶体的剪切模量 $(\mathrm{Pa})$; $B$ 为 Burgers 矢量大小 $(\mathrm{m}) ; \gamma$ 为晶体的比表面自由能 $\left(\mathrm{J} \cdot \mathrm{m}^{-2}\right)$ 。

$6 \mathrm{H}-\mathrm{SiC}$ 单晶原生小面处的微管结构如图 5 所示, 测试范围 $60 \mu \mathrm{m} \times 60 \mu \mathrm{m}$ 。由图 5 可知, 晶体生长螺 旋台阶都是从深红色的圆点处产生，这些深红色的 圆点就是微管缺陷在晶体原生表面的露头点。对于 AFM 测试结果来说, 每幅图右侧配有相应的高度值, 深色代表位置相对低的区域，而浅色代表位置相对 高的区域。虽然微管是空心管，但是由于 AFM 测试 方法的限制，微管呈现深色的圆孔。经 AFM 测试与 统计分析, 得知晶体原生小面处的微管直径分布在 $760 \mathrm{~nm} \sim 6.0 \mu \mathrm{m}$ 之间。

从图 5 还可清晰地看到, 微管周围伴随着至少 一个螺旋台阶, 且螺旋台阶有两种旋向: 左旋(即顺 时针旋转)和右旋(即逆时针旋转)。一个微管缺陷可 以延伸出同样旋向的或不同旋向的螺旋台阶。

\section{2 结果与讨论}

\section{$2.14 \mathrm{H} 、 6 \mathrm{H}-\mathrm{SiC}$ 单晶原生小面台阶形貌差异} 物理气相传输法生长的 $\mathrm{SiC}$ 单晶, $4 \mathrm{H}-\mathrm{SiC}$ 原生 小面扩展后, 其周边趋向于形成六边形的生长台阶;
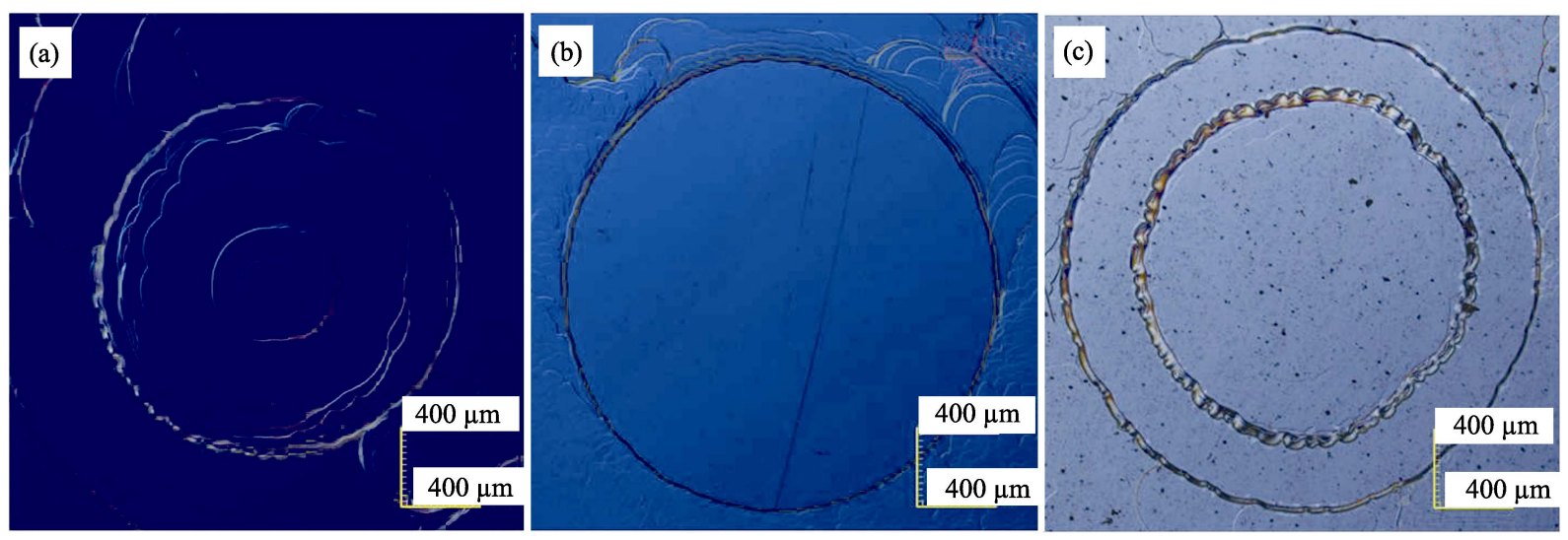

图 4 其他 $6 \mathrm{H}-\mathrm{SiC}$ 单晶原生面形貌

Fig. 4 Surface morphologies of the other as-grown $6 \mathrm{H}-\mathrm{SiC}$ single crystal 


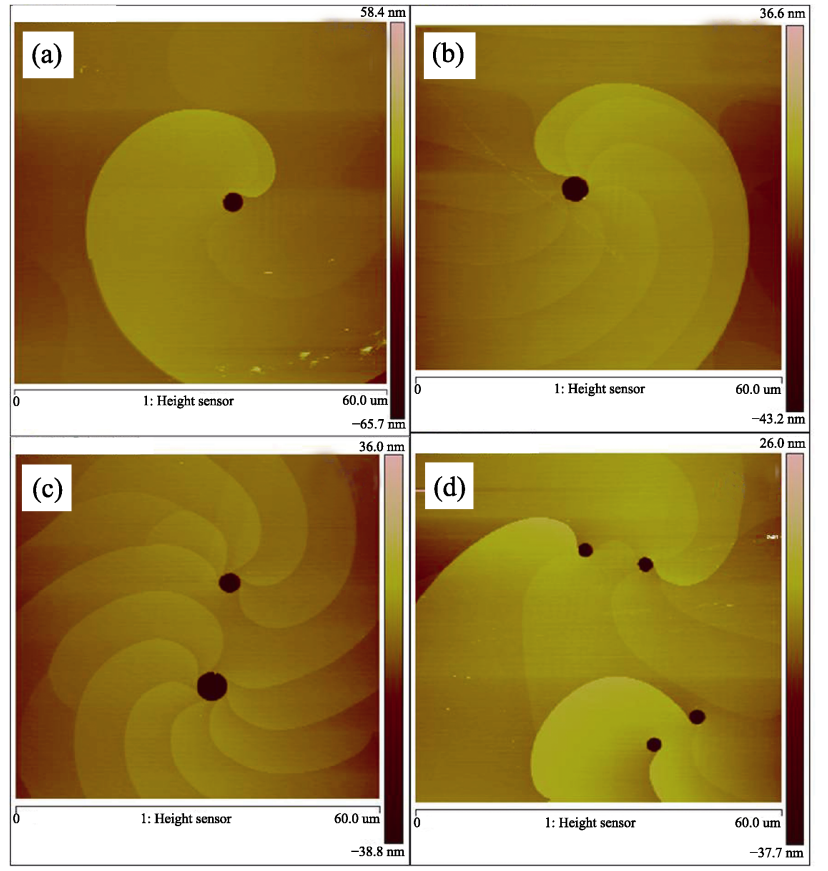

图 $56 \mathrm{H}-\mathrm{SiC}$ 单晶原生小面微管 AFM 照片

Fig. 5 AFM images showing typical micropipes on the facet of a $6 \mathrm{H}-\mathrm{SiC}$ single crystal

而 $6 \mathrm{H}-\mathrm{SiC}$ 原生小面扩展后, 其周边趋向于形成圆 形的生长台阶，这一形貌的差异来源于晶体本身的 内部结构以及生长环境两个因素。

由 Jackson 双层界面模型可知 ${ }^{[10,15]}$, 螺旋生长 台阶是圆形还是多边形主要取决于螺旋台阶的粗粘 程度, 如果台阶是粗粘的, 即扭曲密度大, 生长速 度快, 它在生长前进的过程中不受晶面上结晶方位 各向异性的影响, 呈现为圆形; 反之, 如果台阶比 较光滑, 即扭曲密度小, 生长速度慢, 台阶的生长 前进受到晶面上的结晶方位各向异性的影响, 台阶 呈现为六边形。Jackson 因子 $\alpha$ 如下所示:

$$
\alpha=\frac{\Delta H_{m}}{k T_{m}}\left(\frac{\eta}{v}\right)
$$

其中 $\Delta H_{m} / T_{m}=\Delta S_{\mathrm{f}}$ 为单个原子的熔融熵, $k$ 为波 尔兹曼常数, $\eta$ 为界面上的配位数, $v$ 为晶体内部原 子配位数。也就是说, 固-液生长界面结构是粗粘还 是光滑主要取决于物质的热力学性质, 故按 Jackson 模型, 从热力学角度分析 $4 \mathrm{H} 、 6 \mathrm{H}-\mathrm{SiC}$ 单晶 生长的 Jackson 因子 $\alpha$ 。
在 $\mathrm{Si}-\mathrm{C}$ 系中包含了许多气相物种，根据文献 ${ }^{[16]}$ 给出物质 $\mathrm{SiC} 、 \mathrm{Si} 、 \mathrm{SiC}_{2}$ 的热力学参数焓与温度的 函数关系，见表 1 。其中, $H^{\circ}-H_{298}^{\circ}=4.18 \times(A \times t+$ $\left.B \times t^{2} / 2+C \times t^{3} / 3+D \times t^{4} / 4-E / t+F-H\right), H_{T}^{\circ}$ 是温度 $T$ 下的标准生成焓 $(\mathrm{kJ} / \mathrm{mol})$, 公式中的 $t=$ 温度 $T(\mathrm{~K}) /$ 1000 。

按照表 1 , 以 $\mathrm{Si}(\mathrm{g})+\mathrm{SiC}_{2}(\mathrm{~g})=2 \mathrm{SiC}(\mathrm{s})$ 为例, 设 定 $4 \mathrm{H}-\mathrm{SiC}$ 单晶生长温度为 $2100^{\circ} \mathrm{C}, 6 \mathrm{H}-\mathrm{SiC}$ 单晶生 长温度为 $2200^{\circ} \mathrm{C}$, 通过计算得出 $4 \mathrm{H} 、 6 \mathrm{H}$ 单晶生长 温度下的反应焓分别为 $871.9 \mathrm{~kJ} / \mathrm{mol}$ 和 $873.5 \mathrm{~kJ} / \mathrm{mol}$ 。 同时由 $\mathrm{SiC}$ 单晶结构层可知: $4 \mathrm{H} 、 6 \mathrm{H}-\mathrm{SiC}$ 单晶原生 小面分别是碳原子密排层和硅原子密排层, 碳原子 密排层中任意一个碳原子将与一个最近邻硅原子密 排层中的三个硅原子相连接, 同样地, 硅原子密排 层中任意一个硅原子将与一个最近邻碳原子密排层 中的三个碳原子相连接。故对于 $4 \mathrm{H} 、 6 \mathrm{H}-\mathrm{SiC}$ 单晶 而言, $\eta / v$ 均是 $3 / 4$ 。将上述参数分别带入公式(2)中 得到 $4 \mathrm{H} 、 6 \mathrm{H}-\mathrm{SiC}$ 单晶的 Jackson 因子 $\alpha$ 分别为 33.15 和 $31.87,4 \mathrm{H}-\mathrm{SiC}$ 单晶的 $\alpha$ 大于 $6 \mathrm{H}-\mathrm{SiC}$ 。

综上所述, $4 \mathrm{H} 、 6 \mathrm{H}-\mathrm{SiC}$ 单晶原生小面生长台阶 形貌的差异是由生长界面的粗糙程度引起的。对 $4 \mathrm{H} 、 6 \mathrm{H}-\mathrm{SiC}$ 单晶而言, 单晶生长温度是决定 Jackson 因子大小的关键参数, 也就是说, 单晶生长温度的 差异是 $4 \mathrm{H} 、 6 \mathrm{H}-\mathrm{SiC}$ 单晶原生小面生长台阶形貌差 异的本质因素。晶体在气相中的生长与在液相生长 中时 Jackson 因子 $\alpha$ 意义一样, 即 $\alpha$ 越大阶梯扭曲密 度越小, 生长表面越平滑, 螺旋生长层的形状越易 呈现多边形。

\section{2 原生小面微管结构}

图 6 是图 5(b)原生小面微管缺陷的 AFM 三维照 片, 由三维立体结构可知: 以微管为中心散发出 7 条螺旋生长台阶, 该螺旋台阶的相对高度随着台阶 至微管中心的距离变化而变化。由图 7 台阶截面图 可知螺旋台阶的相对高度, 有的螺旋台阶相对较高, 对应较大的伯格斯矢量; 有的螺旋台阶相对较低, 对应较小的伯格斯矢量。对微管的直径、螺旋台阶 高度以及旋向进行统计分析, 结果如表 2 所示。现 规定：对于右旋螺旋台阶，逆时针方向测量台阶高 度; 对于左旋螺旋台阶，顺时针方向测量台阶高度。

表 $1 \mathrm{SiC} 、 \mathrm{Si} 、 \mathrm{SiC}_{2}$ 的焓与温度的函数关系

Table 1 Enthalpy of $\mathrm{SiC}, \mathrm{Si}, \mathrm{SiC}_{2}$ as a function of temperature

\begin{tabular}{cccccccccc}
\hline Species & $H_{298}$ & $\mathrm{~A}$ & $\mathrm{~B}$ & $\mathrm{C}$ & $\mathrm{D}$ & $\mathrm{E}$ & $\mathrm{F}$ & $\mathrm{G}$ & $\mathrm{H}$ \\
\hline $\mathrm{Si}$ & 0 & & & & & & & & \\
$\mathrm{SiC}$ & 718.96 & 7.970 & 1.06 & -0.210 & 0.0138 & 0.73 & 171.470 & 62.757 & 172 \\
$\mathrm{SiC}_{2}$ & 614.46 & 17.314 & -3.23 & 1.256 & -0.1150 & -1.24 & 138.586 & 73.640 & 147 \\
\hline
\end{tabular}




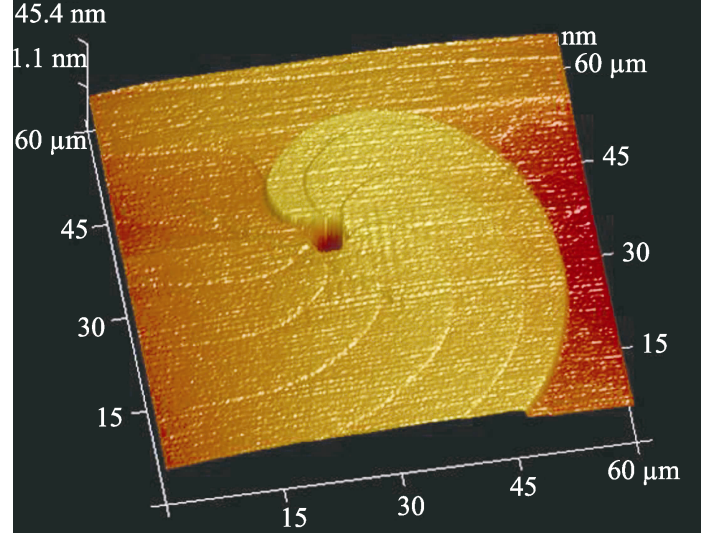

图 6 原生小面微管 3D-AFM 形貌

Fig. 6 Three dimensional AFM image showing typical micropipes on the facet of a $6 \mathrm{H}-\mathrm{SiC}$ single crystal

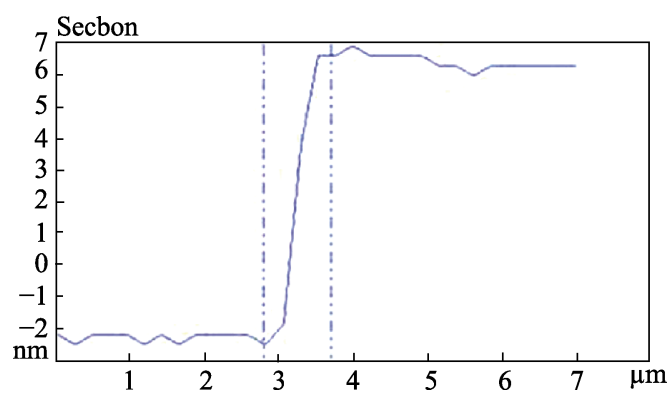

图 7 原生小面微管台阶截面图

Fig. 7 Section image of the spiral step on the as-grown surface of single crystal

表 2 微管直径与螺型台阶高度之间的关系 Table 2 Relationship between micropipe diameter and the screw type step height

\begin{tabular}{ccccccc}
\hline Micropipe & 1 & 2 & 3 & 4 & 5 & 6 \\
\hline $\begin{array}{c}\text { Diameter/ } \mu \mathrm{m} \\
\text { The number }\end{array}$ & 3.1 & 4.5 & 2.5 & 2.5 & 2.6 & 2.6 \\
$\begin{array}{c}\text { of steps } \\
\text { Step height/nm }\end{array}$ & -12.0 & 7 & 5 & 3 & 2 & 1 \\
& -1.9 & 1.6 & -6.0 & -2.1 & -1.1 & \\
& & 1.3 & -1.3 & -2.2 & & \\
& & 1.6 & -1.6 & & & \\
& & 1.6 & -1.9 & & & \\
$B / \mathrm{nm}^{2}$ & & 1.3 & & & & \\
$\left(D / B^{2}\right) / \mathrm{nm}^{-1}$ & 17.0 & 13.9 & 17.4 & 11.1 & 23.6 & 23.6 \\
\hline
\end{tabular}

由微管结构的统计分析可知:

(1) 微管直径分布在 $760 \mathrm{~nm}$ 6.0 $\mu \mathrm{m}$ 之间;

(2) 微管的伯格斯矢量绝对值分布在 $5 b_{0} \sim 14 b_{0}$ $\left(\mathrm{b}_{0}=\mathrm{c}=1.5079 \mathrm{~nm}\right)$;

(3) 微管周围的螺旋台阶可以全部由左螺旋组 成或全部由右螺旋组成，也可以是左右螺旋共同组
成，但大多数都是同样旋向的;

(4) $D / B^{2}$ 分布在 $11.1 \sim 23.6 \mathrm{~nm}^{-1}$ 之间, 即获得的 微管结构数据不严格遵守 Frank 理论。

微管结构不严格遵守 Frank 理论有如下原因:

(1) AFM 测试误差, 由于台阶呈现螺旋单调上 升结构，同一螺旋台阶不同位置的台阶高度略有 差距;

（2）有研究表明构成微管的位错包含螺位错、 混合型位错等不同类型 ${ }^{[17]}$ ，而 AFM 获得的台阶高 度信息只能代表位错的螺型分量的大小，而无法反 映混合型位错真实的伯格斯矢量大小。

\section{3 结论}

本研究对物理气相传输法生长的 $4 \mathrm{H} 、 6 \mathrm{H}-\mathrm{SiC}$ 单晶原生小面的表面形貌进行了观察和研究，同时 在 Jackson 模型下，从热力学角度计算了 $4 \mathrm{H}$ 、 $6 \mathrm{H}-\mathrm{SiC}$ 单晶的 Jackson 因子 $\alpha$ 分别为 33.15 和 31.87 。 故 $4 \mathrm{H} 、 6 \mathrm{H}-\mathrm{SiC}$ 单晶原生小面台阶形貌的差异是生 长界面的粗糖程度和单晶生长温度引起的。

原生小面的微管缺陷是生长台阶的生长源，以 微管为中心散发出数条螺旋生长台阶。微管直径分 布在 $760 \mathrm{~nm} \sim 6.0 \mu \mathrm{m}$ 之间; 微管直径与伯格斯矢量 平方值的商 $D / B^{2}$ 分布在 $11.1 \sim 23.6 \mathrm{~nm}^{-1}$ 之间, 即获 得的微管结构数据不严格遵守 Frank 理论。

\section{参考文献:}

[1] HIROYUKI MATSUNAMI. Technological breakthroughs in growth control of silicon carbide for high power electronic devices. Japanese Journal of Applied Physics, 2004, 43(10): 6835-6847.

[2] KARL O DOHNKE, KARSTEN GUTH, NICOLAS HEUCK. History and recent developments of packaging technology for $\mathrm{SiC}$ power deveces. Materials Science Forum, 2016, 858: 1043-1048.

[3] XUN QIAN, XUN BOYANG, LI ZUXIN. Application of SiC power electronic devices in secondary power source for aircraft. Renewable and Sustainable Energy Reviews, 2017, 70: 1336-1342.

[4] ZHANG YU, CHEN HUI, CHOI GLORIA, et al. Nucleation mechanism of $6 \mathrm{H}-\mathrm{SiC}$ inclusions inside 15R-SiC crystals. Journal of Electronic Materials, 2010, 39(6): 799-804.

[5] MASATOSHI KANAYA, JUN TAKAHASHI, YUIHIRO FUJIWARA, et al. Controlled sublimation growth of single crystalline $4 \mathrm{H}-\mathrm{SiC}$ and $6 \mathrm{H}-\mathrm{SiC}$ and identification of polytypes by X-ray diffraction. Appl. Phys. Lett, 1991, 58(1): 56-58.

[6] KIM JUN GYU, JEONG JIN HWAN, KIM YOUNGHEE, et al. Evaluation of the change in properties caused by axial and radial temperature gradients in silicon carbide crystal growth using the 
physical vapor transport method. Acta Materialia, 2014, 77: 54-59.

[7] STEINK R A, LANIG P. Control of polytype formation by surface energy effects during the growth of $\mathrm{SiC}$ monocrystals by the sublimation method. Journal of Crystal Growth, 1993, 131(71): 71-74.

[8] NORBERT SCHULZE, DONOVAN L BARRETT, GERHARD PENSL, et al. Near-thermal equilibrium growth of $\mathrm{SiC}$ by physical vapor transport. Materials Science and Engineering B, 1999, 61-62: $44-47$.

[9] STRAUBINGER T L, BICKERMANN M, HOFMANN D, et al. Stability criteria for $4 \mathrm{H}-\mathrm{SiC}$ bulk growth. Materials Science forum, 2001, 353-356: 25-28.

[10] 王文魁, 王继扬, 赵珊茸. 晶体形貌学. 武汉: 中国地质大学出 版社, 2001: 1-5.

[11] KAZUMA ETO, HIROMASA SUO, TOMOHISA KATO, et al. Growth of p-type $4 \mathrm{H}-\mathrm{SiC}$ single crystals by physical vapor transport using aluminum and nitrogen co-doping. Journal of Crystal
Growth, 2017, 470: 154-158.

[12] NORIHIRO HOSHINO, ISAHO KAMATA, YUICHIRO TOKUDA, et al. Fast growth of n-type $4 \mathrm{H}-\mathrm{SiC}$ bulk crystal by gas-source method. Journal of Crystal Growth, 2017, 478: 9-16.

[13] FRANK F C. Capillary equilibria of dislocated crystals. Acta Cryst., 1951, 4: 497-501.

[14] SANDEEP MAHAJAN, ROKADE M Y, ALI S T, et al. Investigation of micropipe and defects in molten $\mathrm{KOH}$ etching of $6 \mathrm{H}$ n-silicon carbide single crystal. Materials Letters, 2013, 101: 72-75.

[15] 刘全坤, 祖方遒, 孙国雄, 等. 材料成形基本原理. 北京: 机械 工业出版社, 2009: 67-71.

[16] DONG JIE, LIU ZHE, XU XIANGANG, et al. Investigation on the thermodynamics and kinetics of the growth of $\mathrm{SiC}$ single crystal. Journal of Synthetic Crystals, 2004, 33(3): 283-287.

[17] HEINDL J, DORSCH W, STRUNK H P. Dislocation content of micropipes in SiC. Physical Review Letters, 1998, 80(4): 740741. 